\title{
Awake Examination Versus DISE for Surgical Decision Making in Patients With OSA: A Systematic Review
}

\author{
Victor F. Certal, MD, PhD; Rui Pratas, MD; Lidia Guimarães, MD; Rodolfo Lugo, MD; Yungan Tsou, MD; \\ Macario Camacho, MD; Robson Capasso, MD
}

\begin{abstract}
Objective: Traditionally, upper airway examination is performed while the patient is awake. However, in the past two decades, drug-induced sleep endoscopy (DISE) has been used as a method of tridimensional evaluation of the upper airway during pharmacologically induced sleep. This study aimed to systematically review the evidence regarding the usefulness of DISE compared with that of traditional awake examination for surgical decision making in patients with obstructive sleep apnea (OSA).

Data Sources: Scopus, PubMed, and Cochrane Library databases were searched.

Review Methods: Only studies with a primary objective of evaluating the usefulness of DISE for surgical decision making in patients with OSA were selected. The included studies directly compared awake examination data with DISE outcome data in terms of possible influences on surgical decision making and operation success.

Results: A total of eight studies with 535 patients were included in this review. Overall, the surgical treatment changed after DISE in 50.24\% (standard deviation 8.4) cases. These changes were more frequently associated with structures contributing to hypopharyngeal or laryngeal obstruction. However, these differences do not automatically indicate a higher success rate.

Conclusion: This review emphasized the direct impact of DISE compared with that of awake examination on surgical decision making in OSA patients. However, it is also clear that the available published studies lack evidence on the association between this impact and surgical outcomes.
\end{abstract}

Key Words: DISE, obstructive sleep apnea.

Laryngoscope, 126:768-774, 2016

\section{INTRODUCTION}

Sleep-disordered breathing (SDB) is a broad term that encompasses, among others, snoring, upper airway resistance (UAR) syndrome, and obstructive sleep apnea (OSA).

Obstructive sleep apnea syndrome is a common disorder, with a prevalence of $2 \%$ to $4 \%$ in the adult population and an increasing rate of morbidity and mortality. ${ }^{1}$ Pharyngeal collapse during sleep as a consequence of abnormal structural anatomy and loss of muscle tone dur-

From the CINTESIS (Center for Research in Health Technologies and Information Systems), University of Porto (v.F.c.); the Department of Otorhinolaryngology/Sleep Medicine Center, Hospital CUF and Hospital Sao Sebastiao (v.f.c., R.P., L.G.), Porto, Portugal; the Department of Otorhinolaryngology, Grupo Medico San Pedro (R.L.), Monterrey, Mexico; the Department of Otolaryngology, Division of Sleep Surgery and Medicine, China Medical University Hospital (ч.т.), Taichung, Taiwan; the Department of Otolaryngology, Division of Sleep Surgery and Medicine, Tripler Army Medical Center (M.c.), Honolulu, Hawaii; and the Sleep Surgery Division, Department of Otolaryngology-Head and Neck Surgery, Stanford University School of Medicine (R.c.), Stanford, California, U.S.A.

Editor's Note: This Manuscript was accepted for publication September 16, 2015.

The authors have no funding, financial relationships, or conflicts of interest to disclose.

Send correspondence to Victor Certal, MD, PhD, Hospital CUFPorto, Serviço de Otorrinolaringologia, Estrada da Circunvalação 14341, 4100-180 Porto, Portugal. E-mail: victorcertal@gmail.com

DOI: 10.1002/lary.25722 ing sleep are characteristic of OSA, with snoring being a cardinal sign. ${ }^{2}$

Fundamentally, continuous positive airway pressure (CPAP) therapy remains the gold standard for the conservative treatment of OSA; however, surgery may be indicated to improve compliance and outcomes in patients with poor tolerance to $\mathrm{CPAP}^{3,4}$ Increasing recognition of the multilevel nature of anatomical obstruction consequentially indicates the existence of a large variety of differing surgical techniques used in an attempt to address this problem. ${ }^{5}$ Currently, there is no consensual gold standard method to determine the level of airway collapse. Traditionally, upper airway examination is performed while the patient is awake. However, in the past two decades, drug-induced sleep endoscopy (DISE) has been used as a method of tridimensional evaluation of the upper airway during pharmacologically induced sleep. ${ }^{6}$ Several studies have attempted to demonstrate the usefulness of DISE as a diagnostic approach to sleep apnea, proving that the method is simple, safe, and cost-effective. ${ }^{7-9}$ However, data on the comparison of DISE with awake examination in terms of the usefulness for surgical decision making remains sparse.

In this study, we systematically reviewed evidence regarding the usefulness of DISE compared with that of 
traditional awake examination for surgical decision making in patients with OSA.

\section{MATERIALS AND METHODS}

\section{Search Strategy}

A comprehensive literature search was initially performed in January 2015, followed by an update in May 2015, using the electronic databases of the Cochrane Library, SCOPUS, and PubMed. We combined the following keywords: "Sleep Endoscopy"; "DISE"; "Drug-Induced Sleep Endoscopy"; "SleepDisordered Breathing"; and "Sleep Apnea." No language restrictions were applied. Bibliographies of all selected articles and review articles were also reviewed to find any other relevant article. To minimize the risk of missing relevant data, we also searched abstracts and conference proceedings of relevant congresses and scientific forums from 2011 to 2014 by hand.

\section{Selection Criteria, Study Quality Assessment, and Data Analysis}

Only studies with a primary objective of evaluating the usefulness of DISE for surgical decision making in patients with OSA were selected. The included studies directly compared awake examination data with DISE outcome data in terms of possible influences on surgical decision making. Studies in which all included patients did not undergo both examinations, those in which the impact on surgical decision making was not clearly stated, and those that focused on pediatric populations were excluded.

Data were abstracted by two independent reviewers (R.P. and L.G.) in a blinded manner, and discrepancies were resolved by a third reviewer (v.F.C.). Two authors (M.c. and R.c.) also independently assigned the 14-item Quality Assessment Tool for Diagnostic Accuracy Studies (QUADAS) to each article. Disagreements were resolved by consensus. The primary outcome was evidence regarding the usefulness of DISE compared with that of traditional awake examination for surgical decision making in patients with OSA. The secondary outcome measures included the impact of DISE on surgical outcomes and all other outcomes described in the included studies. Data were reported as mean/standard deviation (SD) (range) values for continuous variables and frequency and percentage values for categorical variables.

\section{RESULTS}

\section{Search Results and Quality Assessment}

All database searches were performed in January 2015, with an update in May 2015. A flow chart of the process of study identification and inclusion/exclusion is shown in Figure 1.

In total, 393 articles were identified using the search strategy and listed sources. After the titles and abstracts were screened for relevance, 363 articles were excluded (the reasons for exclusion are presented in Fig. 1). The remaining 30 articles were retrieved for more detailed full-article evaluation, and 23 were excluded because of the lack of a direct comparison between awake examination and DISE, or because of the lack of mention to the impact on surgical decision making. ${ }^{10-32}$ One study ${ }^{33}$ was included after a manual search of references of the included studies. Finally, eight studies were included in this review (Table I) ${ }^{5,33-39}$

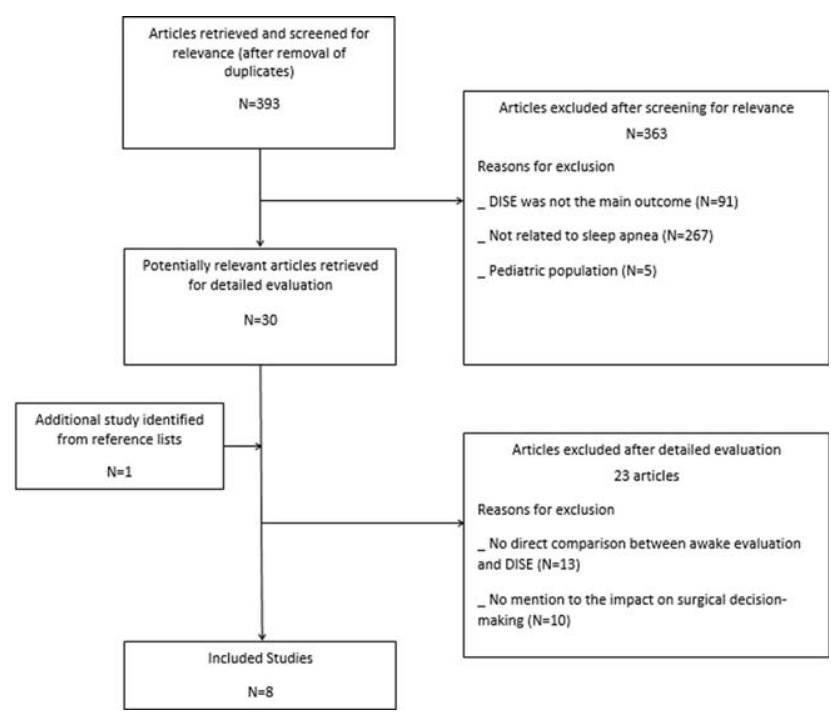

Fig. 1. Flow diagram of study identification and selection.

The QUADAS checklist for the assessment of methodological quality is presented in Table II. Overall, the included studies satisfied seven of the 14 items in the QUADAS checklist, and the primary methodological limitations of the studies were related to poor reporting of items 3, 4, and 13 .

\section{Included Studies}

A total of eight studies with 535 patients were included in this review. The details and main results of these articles are summarized in Table I. Sleep was induced using intravenous propofol in five studies, ${ }^{5,34,36,38,39}$ a combination of propofol and midazolam in two, ${ }^{33,37}$ and intravenous midazolam in one. ${ }^{35}$

Five studies $^{33,35-38}$ analyzed whether surgical planning after DISE was different from that after awake clinical examination. Overall, the surgical treatment changed after DISE in $50.24 \%$ cases (SD 8.4). These changes were more frequently associated with structures contributing to hypopharyngeal or laryngeal obstruction. The frequency of multilevel airways collapse detected by DISE was greater than that detected by awake examination.

Three studies analyzed the correlation between the modality used for surgical planning (awake evaluation or DISE) and surgical outcomes. ${ }^{5,34,39}$ Aktas et al. ${ }^{5}$ found that the obstruction site defined by DISE was correlated with increased surgical success; specifically, a higher surgical success rate for cases of upper airway obstruction and a lower surgical success rate for cases of lower airway obstruction. There was no correlation between awake examination and surgical outcomes. In contrast, Yilmaz et al. ${ }^{39}$ did not find any correlation between DISE and surgical success, despite the higher rate of combined procedures performed in those subjects who underwent DISE when compared with those who underwent only during awake examination (in this case, the Müeller maneuver). However, that study included only patients with retropalatal obstruction. Finally, Blumen et al. ${ }^{34}$ analyzed the reliability of DISE by studying OSA 


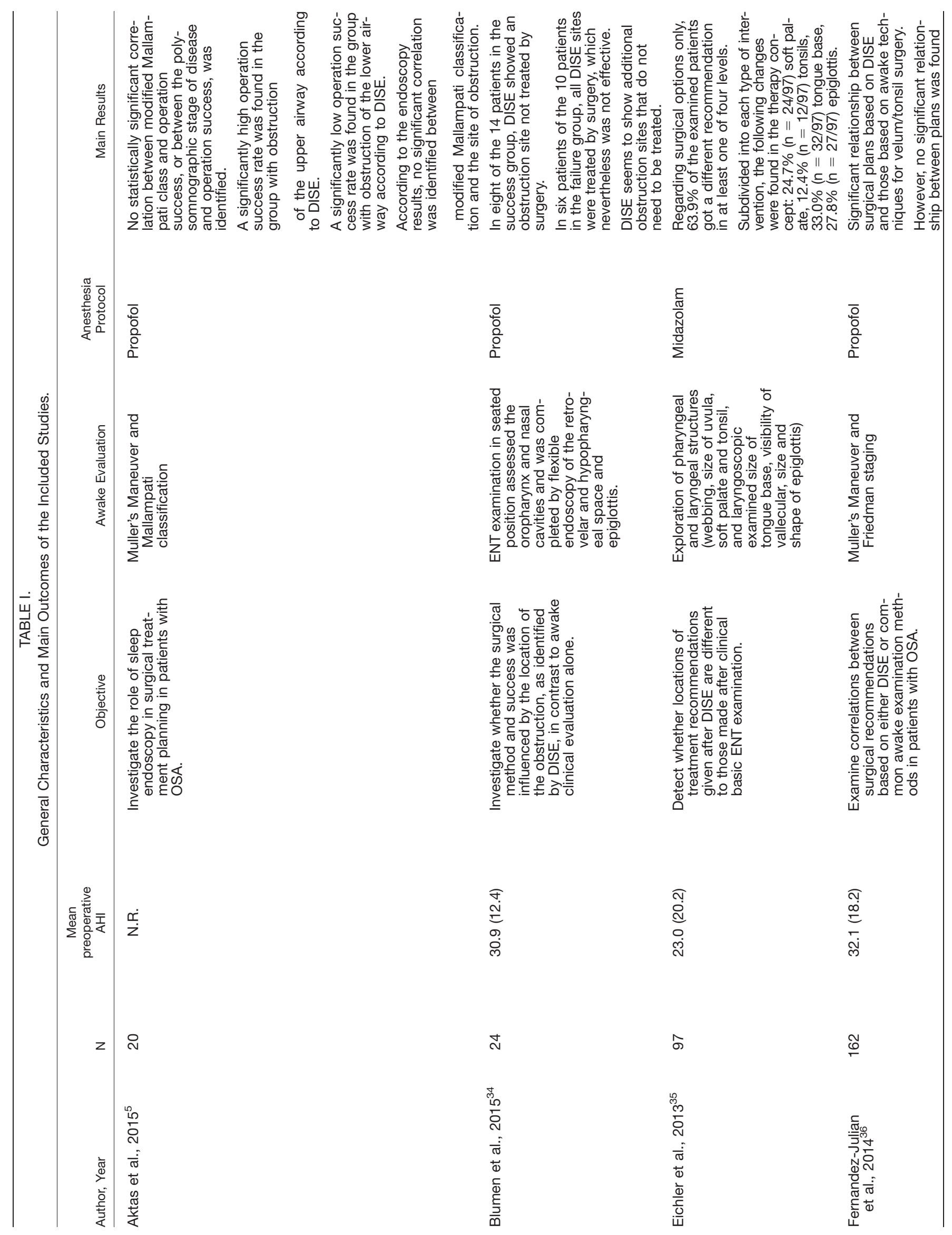




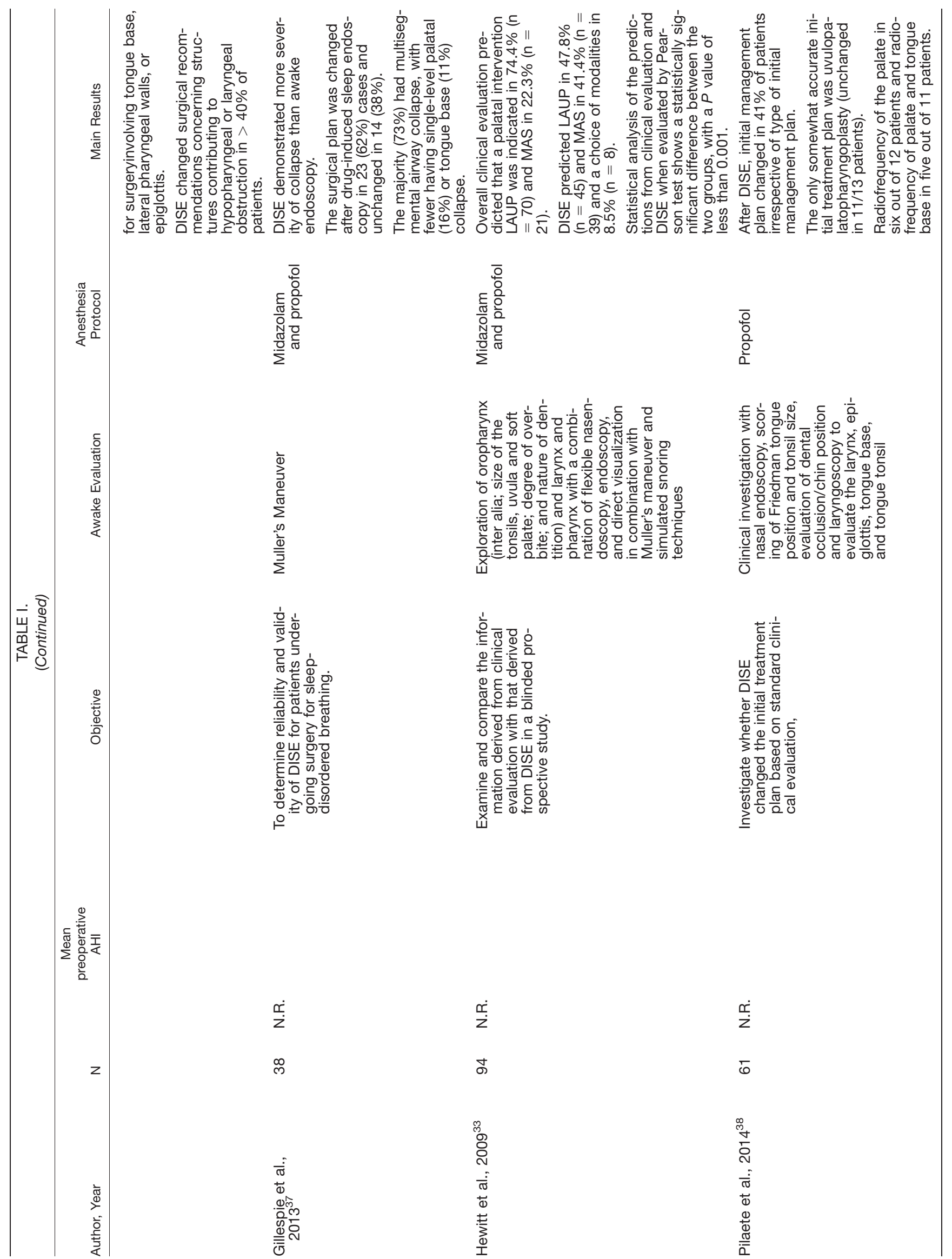


patients with surgical indications identified during awake examination; DISE before surgery identified obstruction sites that were either the same as or different from those found during awake examination. Therefore, the surgical procedures based on the latter were either complete or partial when the DISE findings were considered. The proportion of concentric retropalatal obstruction and retrolingual obstruction cases was higher in the failure group, indicating that DISE could have some utility in predicting bad surgical outcomes. However, the success group included cases in which DISE had identified retrolingual or epiglottic obstruction sites that were not operated, although the treatment was still effective, suggesting that DISE may show collapse sites that may not be relevant to individual patients.

\section{DISCUSSION}

Polysomnography is the gold standard diagnostic tool for SDB and is essential for the evaluation of OSA severity. However, it cannot localize the sites and patterns of upper airway obstruction. Awake upper airway evaluation is a routine procedure, but its role is frequently controversial because it is performed in the awake state wherein an increased muscle tone can confound the results. In 1991, Croft and Pringle ${ }^{40}$ introduced the technique of sleep endoscopy wherein the upper airway was examined during midazolam-induced sleep. The possible obstruction sites could be visualized using a flexible camera inserted through the nasal cavity in an anesthetic-induced sleep state, and surgery was planed according to the findings.

Several studies ${ }^{27,28}$ have reported a discrepancy between obstruction sites detected during awake examination and those detected during sleep endoscopy. However, few studies have investigated the actual impact of these discrepancies on surgical decision making.

The primary conclusion of the present review is that surgical planning performed according to the findings of awake examination can change markedly after DISE ( $>50 \%$ cases), and the differences are most frequently associated with the hypopharyngeal and laryngeal structures. However, these differences do not automatically indicate a higher success rate. Very few studies considered the actual implications of DISE for surgical success, and the results of these studies are heterogeneous. Therefore, a solid consensus could not be reached by analyzing the literature. Furthermore, Blumen et al. ${ }^{34}$ showed that the treatment of all obstructed sites detected by DISE is not a guarantee of success, and additional sites detected by DISE indeed may lead to unnecessary procedures. High-drug doses and prolonged examination, oversensitive observation, misunderstanding of the correlation between the DISE image and considerably decreased airflow, and induction of secondary obstruction sites by the primary sites may account for these potentially artificial additional sites.

The ideal anesthetic for use during DISE is another controversial issue. Although propofol and/or midazolam have been the drugs of choice in the included studies, the search persists for a drug that alters sleep 
TABLE II.

Assessment of the Methodological Quality of the Studies Included According to the 14-item QUADAS Checklist.*

\begin{tabular}{|c|c|c|c|c|c|c|c|c|c|c|c|c|c|c|}
\hline \multirow[b]{2}{*}{ Author, year, reference } & \multicolumn{14}{|c|}{ Question } \\
\hline & 1 & 2 & 3 & 4 & 5 & 6 & 7 & 8 & 9 & 10 & 11 & 12 & 13 & 14 \\
\hline Aktas et al., $2015^{5}$ & Yes & Yes & Unclear & Unclear & Yes & Yes & Yes & Yes & Yes & Unclear & Unclear & Yes & Unclear & N.A. \\
\hline Blumen et al., $2015^{34}$ & Yes & Yes & Unclear & Yes & Yes & Yes & Yes & Yes & Yes & Unclear & Unclear & Yes & Unclear & N.A. \\
\hline Eichler et al., $2013^{35}$ & Yes & Yes & Unclear & Unclear & Yes & Yes & Yes & Yes & Yes & Yes & Yes & Yes & Unclear & Yes \\
\hline $\begin{array}{l}\text { Fernandez-Julian } \\
\text { et al., } 2014^{36}\end{array}$ & Yes & Yes & Unclear & Unclear & Yes & Yes & Yes & Yes & Yes & Yes & Yes & Yes & Unclear & Yes \\
\hline Gillespie ate al., $2013^{37}$ & Yes & Yes & Unclear & Unclear & Yes & Yes & Yes & Yes & No & Yes & Yes & Yes & Unclear & N.A. \\
\hline Hewitt et al., $2009^{33}$ & Yes & Yes & Unclear & Unclear & Yes & Yes & Yes & Yes & Yes & Yes & Yes & Yes & Unclear & N.A. \\
\hline Pilaete et al., $2014^{38}$ & Yes & Yes & Unclear & Unclear & Yes & Yes & Yes & Yes & Yes & Unclear & Unclear & Yes & Unclear & N.A. \\
\hline Yilmaz et al., $2015^{39}$ & No & Yes & Unclear & Unclear & Yes & Yes & Yes & Yes & Yes & Unclear & Unclear & Yes & Unclear & N.A. \\
\hline
\end{tabular}

${ }^{*}$ QUADAS checklist: 1) Was the spectrum of patients representative of the patients who will receive the test in practice? 2) Were selection criteria clearly described? 3) Is the reference standard likely to classify the target condition correctly? 4) Is the period between reference standard and index test short enough to be reasonably sure that the target condition did not change between the two tests? 5) Did a whole sample or random selection of the sample receive verification using a reference standard? 6) Did patients receive the same reference standard regardless of the index test result? 7 ) Was the reference standard independent of the index test (i.e., the index test did not form part of the reference standard)? 8) Was the execution of the index test described in sufficient detail to permit replication of the test? 9) Was the execution of the reference standard described in sufficient detail to permit its replication? 10) Were the index test results interpreted without knowledge of the results of the reference standard? 11) Were the reference standard results interpreted without knowledge of the results of the index test? 12) Were the same clinical data available when test results were interpreted as would be available when the test is used in practice? 13) Were uninterpretable/intermediate test results reported? 14) Were withdrawals from the study explained?

N.A. = not applicable; QUADAS = Quality Assessment of Diagnostic Accuracy Studies.

architecture to a lesser degree. Recently, dexmedetomidine was advocated as a safer and better choice for DISE. $^{41}$ Dexmedetomidine is a highly selective alpha-2 adrenoreceptor agonist with analgesic and sedative effects and little effect on ventilation. In addition, it is considered to show both cardioprotective and neuroprotective properties. However, despite good preliminary studies, robust evidence supporting its use as a consistent alternative for sleep endoscopy is lacking.

The large heterogeneity in awake examination and DISE procedures in the included studies is a limitation of this review. The low level of evidence in the majority of studies did not allow us to reach firm conclusions regarding several aspects of DISE, thus necessitating more high-level evidence studies. Also, due to the pervasive heterogeneity in multiple areas of the available data, we did not conduct a pooled analysis, and the results of our statistical analysis should be interpreted with caution.

Even without performing a meta-analysis, however, decisions regarding the optimal categorization and analysis of such disparate data necessarily introduce some degree of subjectivity into our analysis.

Because several variables are different between OSA patients (i.e., age, body mass index, prior surgeries, cephalometric variables, sex, race), which contribute to heterogeneity, future studies could consider a randomized block design with a matched pairs analysis. Compared to a completely randomized design, this type of study reduces variability and potential confounding, producing a better estimate of treatment effects in each group. This type of high-level study could assess specifically whether DISE findings are truly related to treatment outcomes.

\section{CONCLUSION}

This review emphasized the direct impact of DISE compared with that of awake examination on surgical decision making in OSA patients and demonstrated that more than $50 \%$ of the surgical planning could be modified after DISE. However, it is also clear that the available published studies lack evidence on the association between this impact and surgical outcomes. Accordingly, it can be concluded that DISE may emerge as an objective tool to anatomically and functionally assess the upper airway in potential surgical OSA patients. However, high-quality evidence level studies with statistically appropriate sample sizes and clinical crossvalidations are necessary to determine the role of DISE in the assessment of treatment outcomes.

\section{BIBLIOGRAPHY}

1. Marin JM, Carrizo SJ, Vicente E, Agusti AG. Long-term cardiovascular outcomes in men with obstructive sleep apnoea-hypopnoea with or without treatment with continuous positive airway pressure: an observational study. Lancet 2005;365:1046-1053.

2. Young T, Peppard PE, Gottlieb DJ. Epidemiology of obstructive sleep apnea: a population health perspective. Am J Respir Crit Care Med 2002:165:1217-1239.

3. Certal V, Nishino N, Camacho M, Capasso R. Reviewing the systematic reviews in OSA surgery. Otolaryngol Head Neck Surg 2013;149:817-829.

4. Camacho M, Riaz M, Capasso R, et al. The effect of nasal surgery on continuous positive airway pressure device use and therapeutic treatment pressures: a systematic review and meta-analysis. Sleep 2015;38:279-286.

5. Aktas O, Erdur O, Cirik AA, Kayhan FT. The role of drug-induced sleep endoscopy in surgical planning for obstructive sleep apnea syndrome. Eur Arch Otorhinolaryngol 2015;272:2039-2043. doi: 10.1007/s00405014-3162-8.

6. De Vito A, Carrasco Llatas M, Vanni A, et al. European position paper on drug-induced sedation endoscopy (DISE). Sleep Breath 2014;18:453-465.

7. Bachar G, Nageris B, Feinmesser R, et al. Novel grading system for quantifying upper-airway obstruction on sleep endoscopy. Lung 2012;190: $313-318$

8. Cho JS, Soh S, Kim EJ, et al. Comparison of three sedation regimens for drug-induced sleep endoscopy. Sleep Breath 2015;19:711-717. doi: 10.1007/s11325-015-1127-9.

9. Carrasco M, Agostini G, Rodrigo A, Giner P, Gomez F, Dalmau J. DISE: a 2-drug comparison and simultaneous polysomnography. Otolaryngol Head Neck Surg 2012;147:P120.

10. Abdullah VJ, Lee DL, Ha SC, van Hasselt CA. Sleep endoscopy with midazolam: sedation level evaluation with bispectral analysis. Otolaryngol Head Neck Surg 2013;148:331-337.

11. Aran X, Gea J, Sauleda J, Aguar MC, Orozco-Levi M, Broquetas JM. [Usefulness of respiratory endoscopy in patients with severe rhonchi and/or 
sleep apnea syndrome]. [Article in Spanish]. Med Clin (Barc) 1994;103: $129-131$

12. Belgu AU, Erdogan B, San T, Gurkan E. The relationship between AHI, Epworth scores and sleep endoscopy in patients with OSAS. Eur Arch Otorhinolaryngol 2015;272:241-245.

13. Borek RC, Thaler ER, Kim C, Jackson N, Mandel JE, Schwab RJ. Quantitative airway analysis during drug-induced sleep endoscopy for evaluation of sleep apnea. Laryngoscope 2012;122:2592-2599.

14. Campanini A, Canzi P, De Vito A, Dallan I, Montevecchi F, Vicini C Awake versus sleep endoscopy: personal experience in 250 OSAHS patients. Acta Otorhinolaryngol Ital 2010;30:73-77.

15. Carrasco Llatas M, Dalmau Galofre J, Lopez Martinez R, Fernandez Martinez S, Vinoles J, Lopez R. [Our findings in the sleep endoscopy exams]. [Article in Spanish]. Acta Otorrinolaringol Esp 2005;56:17-21.

16. Cavaliere M, Russo F, Iemma M. Awake versus drug-induced sleep endoscopy: evaluation of airway obstruction in obstructive sleep apnea/hypopnoea syndrome. Laryngoscope 2013;123:2315-2318.

17. Dalmau J, Carrasco Llatas M, Amoros LI, et al. [Video fiber endoscopy during induced sleep]. [Article in Spanish]. Acta Otorrinolaringol Esp 2002;53:502-504.

18. de Mello-Filho FV, da Silva Junior SN, Faria AC, Garcia LV, Changes of the retrolingual pharynx during the Muller manoeuvre and during sleep in sleep apnoea. J Craniomaxillofac Surg 2014;42:1730-1734.

19. De Vito A, Agnoletti V, Berrettini S, et al. Drug-induced sleep endoscopy: conventional versus target controlled infusion techniques--a randomized controlled study. Eur Arch Otorhinolaryngol 2011;268:457-462.

20. den Herder C, van Tinteren H, de Vries N. Sleep endoscopy versus modified Mallampati score in sleep apnea and snoring. Laryngoscope 2005; 115:735-739.

21. Hamans E, Meeus O, Boudewyns A, Saldien V, Verbraecken J, Van de Heyning P. Outcome of sleep endoscopy in obstructive sleep apnoea: the Antwerp experience. B-ENT 2010;6:97-103.

22. Herzog M, Kellner P, Plossl S, et al. Drug-induced sleep endoscopy and simulated snoring in patients with sleep-disordered breathing: agreement of anatomic changes in the upper airway. Eur Arch Otorhinolar yngol 2015;272:2541-2550. doi: 10.1007/s00405-015-3559-z.

23. Iwanaga K, Hasegawa K, Shibata N, et al. Endoscopic examination of obstructive sleep apnea syndrome patients during drug-induced sleep. Acta Otolaryngol Suppl 2003:36-40.

24. Kezirian EJ. Nonresponders to pharyngeal surgery for obstructive sleep apnea: insights from drug-induced sleep endoscopy. Laryngoscope 2011 121:1320-1326.

25. Koutsourelakis I, Safiruddin F, Ravesloot M, Zakynthinos S, de Vries N. Surgery for obstructive sleep apnea: sleep endoscopy determinants of outcome. Laryngoscope 2012;122:2587-2591.

26. Rabelo FA, Kupper DS, Sander HH, et al. A comparison of the Fujita classification of awake and drug-induced sleep endoscopy patients. Braz $J$ Otorhinolaryngol 2013;79:100-105.
27. Ravesloot MJ, de Vries N. One hundred consecutive patients undergoing drug-induced sleep endoscopy: results and evaluation. Laryngoscope 2011;121:2710-2716.

28. Salamanca F, Costantini F, Bianchi A, Amaina T, Colombo E, Zibordi F Identification of obstructive sites and patterns in obstructive sleep apnoea syndrome by sleep endoscopy in 614 patients. Acta Otorhinolar yngol Ital 2013;33:261-266.

29. Soares D, Folbe AJ, Yoo G, Badr MS, Rowley JA, Lin HS. Drug-induced sleep endoscopy vs awake Muller's maneuver in the diagnosis of severe upper airway obstruction. Otolaryngol Head Neck Surg 2013;148:151156

30. Steinhart H, Kuhn-Lohmann J, Gewalt K, Constantinidis J, Mertzlufft F, Iro H. Upper airway collapsibility in habitual snorers and sleep apneics: evaluation with drug-induced sleep endoscopy. Acta Otolaryngol 2000; 120:990-994.

31. Vanderveken OM, Maurer JT, Hohenhorst W, et al. Evaluation of druginduced sleep endoscopy as a patient selection tool for implanted upper airway stimulation for obstructive sleep apnea. J Clin Sleep Med 2013; 9:433-438.

32. Vroegop AV, Vanderveken OM, Boudewyns AN, et al. Drug-induced sleep endoscopy in sleep-disordered breathing: report on 1,249 cases. Laryngo scope 2014;124:797-802.

33. Hewitt RJ, Dasgupta A Singh A Dutta C, Kotecha BT Is sleep nasendoscopy a valuable adjunct to clinical examination in the evaluation of upper airway obstruction? Eur Arch Otorhinolaryngol 2009;266:691-697.

34. Blumen MB, Latournerie V, Bequignon E, Guillere L, Chabolle F. Are the obstruction sites visualized on drug-induced sleep endoscopy reliable? Sleep Breath 2015;19:1021-1026. doi: 10.1007/s11325-014-1107-5.

35. Eichler C, Sommer JU, Stuck BA, Hormann K, Maurer JT. Does druginduced sleep endoscopy change the treatment concept of patients with snoring and obstructive sleep apnea? Sleep Breath 2013;17:63-68.

36. Fernandez-Julian E, Garcia-Perez MA, Garcia-Callejo J, Ferrer F, Marti F, Marco J. Surgical planning after sleep versus awake techniques in patients with obstructive sleep apnea. Laryngoscope 2014;124:1970-1974.

37. Gillespie MB, Reddy RP, White DR, Discolo CM, Overdyk FJ, Nguyen SA. A trial of drug-induced sleep endoscopy in the surgical management of sleep-disordered breathing. Laryngoscope 2013;123:277-282.

38. Pilaete K, De Medts J, Delsupehe KG. Drug-induced sleep endoscopy changes snoring management plan very significantly compared to standard clinical evaluation. Eur Arch Otorhinolaryngol 2014;271:1311-1319.

39. Yilmaz YF, Kum RO, Ozcan M, Gungor V, Unal A. Drug-induced sleep endoscopy versus Muller maneuver in patients with retropalatal obstruction. Laryngoscope 2015;125:2220-2225. doi: 10.1002/lary.25160.

40. Croft CB, Thomson HG, Samuels MP, Southall DP. Endoscopic evaluation and treatment of sleep-associated upper airway obstruction in infants and young children. Clin Otolaryngol Allied Sci 1990;15:209-216.

41. Li P, Zhou P, Shen P. [Feasibility study for the dexmetomidine utend the drug induced sleep endoscopy]. [Article in Chinese]. Lin Chung Er Bi Yan Hou Tou Jing Wai Ke Za Zhi 2014;28:1151-1154. 